\title{
Infants' sensitivity to kinetic information for three-dimensional object shape
}

\author{
MARTHA E. ARTERBERRY and ALBERT YONAS \\ University of Minnesota, Minneapolis, Minnesota
}

\begin{abstract}
Infant sensitivity to kinetic information specifying three-dimensional object shape was assessed using computer-generated random-dot displays. Four-month-old infants were habituated to displays of an object oscillating about two different axes on alternating trials. Following habituation, the infants were tested for recovery from habituation to a display of the same object and a novel object. Both test displays employed a new axis of rotation. The infants generalized habituation to the same object and increased their looking to the new object. These results provide evidence that infants are sensitive to motion-carried information specifying three-dimensional object shape, since the random-dot displays minimized static information that differentiated the two objects. These findings suggest that, at least by 4 months of age, infants can detect subtle differences in shape from purely kinetic information.
\end{abstract}

Kinetic information for spatial layout and object shape is provided by patterns of optical flow (Gibson, 1966, 1979). Optical flow can be produced by movement of an observer in a static environment, generating the traditional depth cue of motion parallax. In addition, the motion of objects in the environment provides kinetic optical information for their shape and motion in depth. The results of recent studies suggest that infants in the first year of life are able to perceive some aspects of three-dimensional object shape specified by kinetic information (Kellman, 1984; Kellman \& Short, 1985; Owsley, 1983; Shaw, Roder, \& Bushnell, 1986; Yonas, Arterberry, \& Granrud, 1987). The goal of the present study was to extend this work by demonstrating that 4-month-olds can perceive the difference between two rectilinear forms specified by kinetic information in the absence of figural features such as contours and intersections.

Two types of paradigms, both employing a habituation of looking procedure, have been used to investigate infants' sensitivity to kinetic information for threedimensional object shape. One paradigm, the transferacross-depth-cues procedure (Yonas \& Pick, 1975), was used in three investigations. In this procedure, the type of proximal information that specifies the objects changes from the habituation to the test phase. Therefore, researchers can rule out the possibility of infants' respond-

This research was supported by National Institute of Child Health and Human Development Grant HD-16924 awarded to Albert Yonas. The first author was supported by Graduate Fellowship HD-07151-08 to the Center for Research in Learning, Perception, and Cognition from the National Institute of Child Health and Human Development. The authors wish to thank Brenda Hartman, Randy Mason, and Ann Sorknes for their assistance in collecting the data; Kim Pearson and David Rosen for computer programming; and Brad Pillow for helpful comments on an earlier draft. Correspondence and requests for reprints should be sent to Albert Yonas, Institute of Child Development, University of Minnesota, 51 East River Road, Minneapolis, MN 55455. ing to the proximal cues rather than the spatial information they provide. Owsley (1983) habituated 4-month-old infants to kinetic presentations of either a cube or a wedge that oscillated around a vertical axis. Shaw et al. (1986) habituated 4- and 6-month-old infants to shadow-cast displays of a rotating solid rectangle or triangle, whereas Yonas et al. (1987) habituated 4-month-olds to shadowcast presentations of either a rotating wire triangle or a tetrahedron. In all three studies, following habituation, the infants viewed static displays of a new object and the old object. During the test phase, the object's shape was specified by binocular rather than kinetic information. Owsley (1983) and Yonas et al. (1987) found evidence suggesting that 4-month-old infants are able to transfer some aspect of object shape from kinetic to binocular information. In contrast, Shaw et al. (1986) found evidence of this ability in 6-month-olds, but not in 4-month-olds.

An alternative to the transfer-across-depth-cues procedure was used by Kellman (1984) and Kellman and Short (1985). Kellman (1984) habituated 4-month-old infants to a videotape of a rotating object (a wedge or an L-shaped object). Kellman and Short (1985) habituated 4-montholds to a rotating wire figure (containing a $95^{\circ}$ or a $165^{\circ}$ angle). During the habituation phase, the infants viewed the same object undergoing transformations about two different axes. Following habituation, the infants were presented with both a new object and the familiar object, both rotating about a new, third, axis. The infants generalized habituation to the familiar object and looked longer at the new object.

To control for the possibility that the infants were responding to static two-dimensional features of the display, rather than to the three-dimensional shape as specified by the motion-carried information, Kellman (1984), Kellman and Short (1985), and Owsley (1983) included a control group of infants who were habituated to successive static views of the moving object presented in the 
kinetic condition. None of the studies found evidence of shape discrimination based on the static views. Therefore, the investigators concluded that infants were not able to differentiate the two objects based on the static aspects of the displays.

In summary, these previous studies, excluding that of Shaw et al. (1986), provided evidence that 4-month-old infants can perceive some aspect of three-dimensional shape specified by motion-carried information. These studies demonstrated that infants can discriminate between planes and volumes (Yonas et al., 1987) and between rectilinear or near-rectilinear and strongly nonrectilinear objects (Owsley, 1983; Kellman, 1984; Kellman \& Short, 1985). Infants' ability to discriminate between two rectilinear objects specified by kinetic information has not been demonstrated.

One limitation of these studies is that all the displays contained figural information for shape. In addition to kinetic information, the infants were presented with displays containing contours and intersection features. The possibility that the infants were responding to kinetic figural aspects of the display, rather than to three-dimensional object shape, has not been ruled out. By using kinetic random-dot displays similar to those developed by Julesz (1971), the present study provided a test of infants' ability to recover three-dimensional shape specified by purely kinetic information, since these displays do not present lines and intersections to which the infant might respond. In kinetic random-dot displays, figural properties (e.g., edges, intersections) are created by processes operating on optical flow information (Nakayama \& Loomis, 1974).

We presented infants with kinetic computer-generated random-dot displays that, when in motion, appeared to adults as either a complete or an incomplete cube. Schematic drawings of each object and a single static frame of each display are shown in Figure 1. Because the displays were identical in external form, the only information specifying differences in three-dimensional object shape was the motion of the randomly arranged dots.

The possibility that static gradients of texture density could differentiate the two objects was minimized by using displays in which a small number of dots were present in the central region (compare Figures 1C and 1D). Also, the small amount of oscillation minimized texture compression. To demonstrate that the objects could not be discriminated on the basis of static textural properties, before the experiment we presented 10 adults, who had viewed the kinetic displays, with 24 photographs of the two objects shown in Figures $1 \mathrm{C}$ and 1D. The photographs presented representative views of the two displays. The subjects were asked to choose which of the two objects was depicted in each photograph; the mean percent correct $(41 \%)$ was lower than would be expected by chance. In addition, no subject reported that the shapes could be differentiated. Since the two objects could not be discriminated by adults on the basis of two-dimensional static information, we assumed that the same would be
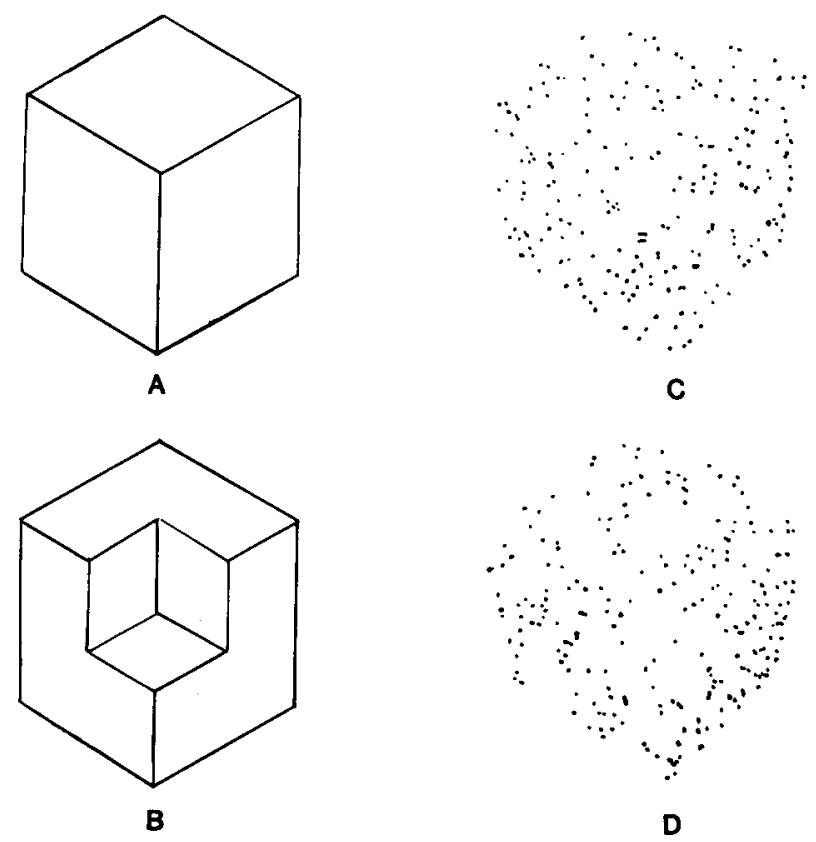

Figure 1. Schematic drawings of the complete (A) and incomplete (B) cubes. These drawings represent the middle frame of each oscillation. $C$ and $D$ depict single frames of the complete and incomplete cube, respectively. In the actual display, the contrast was reversed.

true for infants. Therefore, we did not present infants with static control displays.

In the present study, we used a method similar to the one developed by Kellman (1984). The infants were habituated to displays of the same object oscillating about two different axes. Following habituation, the infants were presented with a display of the same object oscillating about a new, third, axis and a display of a new object oscillating about the new axis. Both test displays present novel directions of dot motion due to the oscillation about a new axis. Thus, this method of presentation allows us to rule out the possibility that infants could dishabituate to a change in a particular pattern of dot velocities traveling in a particular direction.

\section{METHOD}

\section{Subjects}

The subjects were 24 4-month-old infants (mean age 120 days, range 113-135 days). An additional 40 infants began the experiment but did not complete it due to fussiness (34), equipment failure (2), or parent or sibling interruption (4). The high subject attrition may be due to the nature of the displays. The infants exhibited an extraordinarily high level of interest in these displays, and many infants became fussy before showing any evidence of habituation.

\section{Materials and Apparatus}

The infant sat on a parent's lap within a $340 \times 177 \mathrm{~cm}$ threepartitioned surround. The side panels of the surround (each $125 \times 177 \mathrm{~cm}$ ) were oriented approximately $130^{\circ}$ from the front panel. This arrangement provided a homogeneous cubicle that $o c-$ 
cluded the experimental room from the infant's view. The infant sat $65 \mathrm{~cm}$ from a $20 \times 17.5 \mathrm{~cm}$ black-and-white video monitor (subtending $17.5^{\circ} \times 15.3^{\circ}$ of visual angle) that was viewed through an aperture in the front panel of the surround. The experimental room was dark except for light from the monitor and from a $15-\mathrm{W}$ bulb located behind the infant.

There were six displays. The displays consisted of white dots on a black field. Each display simulated an $11-\mathrm{cm}$ complete or incomplete cube undergoing a $30^{\circ}$ rotation around three axes: horizontal, vertical, and diagonal. Schematic line drawings of the two display objects and a depiction of a single frame of each of the objects are shown in Figure I. Figures IA and IB represent the middle frame of all the oscillations, that is, the point at which the object had rotated $15^{\circ}$ of the $30^{\circ}$ oscillation. The missing corner in the incomplete cube was the central, interior corner. The twodimensional arrangement of dots filled a $15 \times 16 \mathrm{~cm}$ area, projecting $13.2^{\circ}$ of visual angle horizontally and $14.0^{\circ}$ of visual angle vertically. The displays were presented on a monitor. Each object was videotaped three times, each time with a different axis of oscillation and a different random arrangement of dots. The luminance of the background was approximately $.5 \mathrm{~cd} / \mathrm{m}^{2}$ and the luminance of the dots was approximately $68 \mathrm{~cd} / \mathrm{m}^{2}$. Each dot was $2.5 \mathrm{~mm}$ in diameter and projected $.2^{\circ}$ of visual angle. Each display contained approximately 240 dots. The displays were generated on an Evans and Sutherland PS300 vector graphics terminal hosted by a VAX computer, and were videotaped from the screen of the computer monitor. The perspective of the displays was chosen to simulate a 3-ft viewing distance. The screen was darkened between trials under control of the experimenter.

The experimenters viewed the infant through $2-\mathrm{cm}$ openings between the front and side panels of the surround. Buttons connected to a laboratory microcomputer were pressed whenever the infant fixated the display. The computer recorded the infant's total fixation time in each trial. In addition, the computer signaled to the second experimenter when each trial had ended and when the infant had reached the habituation criterion.

\section{Procedure}

During the testing session, the infant sat on a parent's lap. The parent's eyes were covered during the experiment, and the parent was instructed not to interact with the infant.

The monitor was dark prior to the beginning of each trial. One experimenter turned on the video recorder to present the display to initiate each trial. The habituation trials followed an infant-control procecture (Horowitz, Paden, Bhana, \& Self, 1972), continuing until the infant looked away from the display for 2 continuous sec or until $60 \mathrm{sec}$ had elapsed. Between trials, the screen was darkened and the tape was changed. After a 5-sec interval, another trial was initiated. This procedure was continued until the infant met the criterion for habituation, which was defined as three consecutive trials with a mean fixation time of less than $50 \%$ of the fixation time from the baseline (the mean of the first three trials). To be included in the baseline, the first trial had to be at least $10 \mathrm{sec}$ in duration. When the habituation criterion was reached, four test trials were presented, using the same infant-control procedure as in the habituation trials.

The infants were habituated, on aiternate trials, to displays of the same object oscillating about two different axes. Following habituation, on alternate trials, they were presented with a display of the habituation object oscillating about a new, third, axis and a display of a new object also oscillating about the new axis. The displays were counterbalanced across infants according to which object and which axes of oscillation were presented during the habituation and test phases. Also, half the infants viewed the novel object first and half viewed the familiar object first in the test phase.

Two experimenters conducted the study. One experimenter, who did not know which stimulus object was being presented on a given trial or whether the infant was in the habituation or test phase of the experiment, observed the infant and recorded fixation times. This experimenter was also responsible for terminating the testing session if the infant became too distressed to continue. Any sibling or parent interference was noted and the data from that subject were not included. The second experimenter controlled the video recorder and changed the displays between trials. To obtain a measure of interjudge reliability, a third experimenter recorded the fixation times of 12 randomly chosen infants. The mean correlation between the fixation times scored by the two observers was $r=.98$. This measure of agreement may be inflated. The fixation durations of the two observers were not independent because the first experimenter's judgments necessarily ended each trial.

\section{RESULTS}

The infants' looking times on the last six habituation trials and on the test trials are presented in Figure 2. For the initial analysis, the looking times on the two novel test trials and the two familiar test trials were combined. The infants' looking times were analyzed in a $2 \times 6 \times 2 \times$ 2 mixed-design analysis of variance with habituation object (complete or incomplete cube), order of presentation of the habituation axes (horizontal, vertical, diagonal), and test order (complete or incomplete cube presented first) as between-subjects factors and novelty (novel and familiar) as a within-subjects factor. The analysis revealed a significant main effect for novelty $[F(1,5)=20.6$, $p<.01]$. No other reliable main effects or interactions were found.

The main effect for novelty indicates that when the data from the two novel test trials and the two familiar test trials are combined, they show that the infants looked significantly longer at the novel object than at the familiar object. Further planned comparisons revealed that infants looked significantly longer at the novel object on the first two test trials $[t(23)=3.38, p<0.01]$, whereas the difference in looking times to the two objects on the last two test trials was not significant.

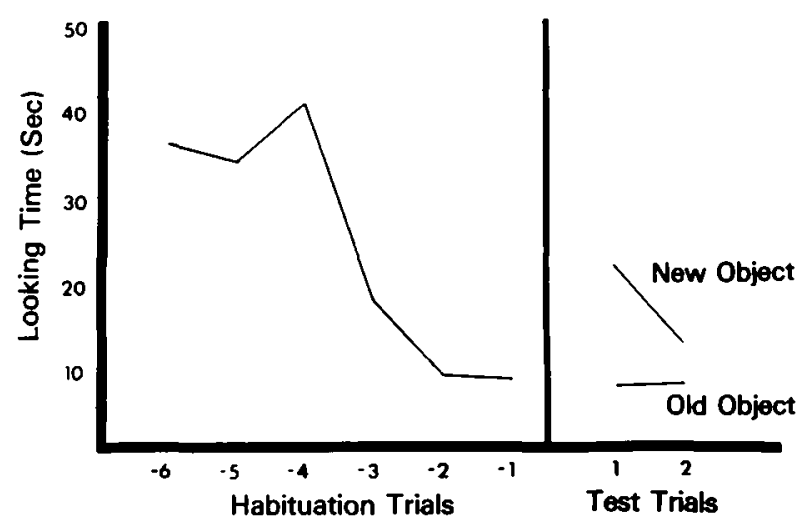

Figure 2. Mean fixation time in habituation and test trials. "OId Object" refers to the same object depicted during the habituntion phase and "New Object" refers to the object not depicted during habituation. 

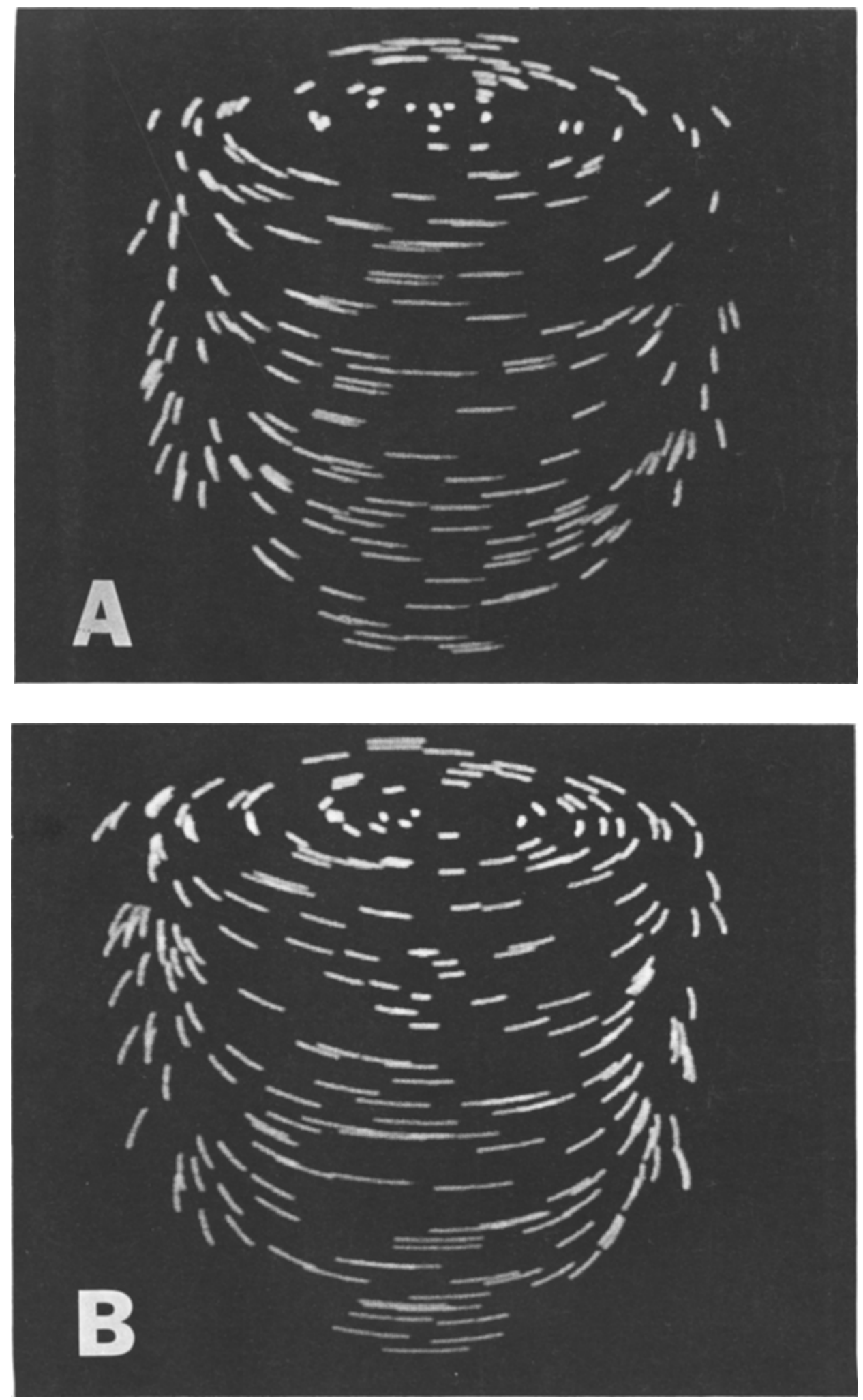

Figure 3. Time-lapse photographs showing the pattern of texture now in the complete (A) and incomplete (B) cube displays. 


\section{DISCUSSION}

The present study is the first to demonstrate that 4month-old infants can discriminate three-dimensional object shape presented by kinetic random-dot displays. In addition, the results of this study suggest that infants are able to discriminate subtle differences, since both objects were rectilinear. Cook and Birch (1984) suggested that shape discrimination in 3-month-olds may be based upon the presence or absence of orthogonality. Caron, Caron, and Carlson (1978, 1979), Owsley (1983), and Kellman (1984) suggested that for infants between 3 and 4 months of age, differences between rectilinear and nonrectilinear objects are quite salient. Although Cook and Birch's (1984) results suggest that discriminations between two rectilinear objects or between two nonrectilinear objects may be more difficult, the present study provides evidence suggesting that by 4 months infants are able to differentiate two rectilinear solids when kinetic information is present.

One limitation of the method used in the present study and in those of Kellman (1984) and Kellman and Short (1985) is that one cannot entirely rule out the possibility that the infants were responding to proximal patterns of stimulation, rather than to the distal shape specified by those patterns. That is, the infants might have discriminated between the patterns of motion that differentiated the two objects without perceiving the three-dimensional object structure. As illustrated in Figure 3 (a time-exposed photograph of two displays), in the upper central region of the complete cube, the distances traveled by the dots are longer than in the equivalent region of the incomplete cube. Although we think this account is less plausible than the assumption that infants perceived the actual threedimensional structure, it cannot be ruled out in this study or in other studies using the transfer-across-axes-ofrotation method developed by Kellman (1984). In addition, static texture density differences could make it logically possible for infants to differentiate the two objects. However, since these objects had similar texture densities and adults were unable to discriminate the two objects based on static information, this possibility seems unlikely. Regardless of whether the infants were responding to the three-dimensional shape or to the twodimensional flow pattern, this study demonstrates rather impressive sensitivity differences in optical motions by 4-month-old infants.

This study also extends our knowledge of infants' abilities to recover information from kinetic random-dot displays. Kinetic dot displays have been employed in a number of recent studies investigating infants' sensitivity to various types of kinetic information. These studies have shown that within the first year of life infants are sensitive to biomechanical motions (Bertenthal, Proffitt, Spetner, \& Thomas, 1985; Fox \& McDaniels, 1982), concurrent motion specifying the approach of a surface (Kellman, Hofsten, \& Soares, 1987), accretion and deletion of texture specifying occlusion (Granrud et al., 1984), and relative motion specifying the ordering of surfaces in depth (Craton \& Yonas, in press). Although all of these studies have shown that infants between 4 and 5 months of age are sensitive to several types of motion-carried information and are able to use this information for perceiving surfaces and relationships between surfaces, the present study is the first to provide evidence that infants can discriminate between the shapes of three-dimensional solid objects presented in kinetic random-dot displays.

The study of young infants' abilities to use motioncarried information for perceiving spatial layouts has just begun. It has been shown that 1 -month-olds are sensitive to optical expansion information for impending collision (Nanez, 1987; Yonas, Pettersen, \& Lockman, 1979) and to optical transformations specifying rigidity or elasticity (Gibson \& Walker, 1984); however, we do not know whether infants at this age are able to perceive threedimensional shape specified by optical flow. There may be more types of information to which infants are sensitive, and it is not known whether sensitivity to different types of information appears simultaneously or whether there is a developmental progression within the general class of kinetic information. Future research should extend the study of the perception of three-dimensional shape from motion to infants younger than 4 months of age.

\section{REFERENCES}

Bertenthal, B. I., Proffitt, D. R., Spetner, N. B., \& Thomas, M. A. (1985). The development of infant sensitivity to biomechanical motions. Child Development, 56, 531-543.

Caron, A., Caron, R., \& Carlson, V. (1978). Do infants see objects or retinal images? Shape constancy revisited. Infant Behavior \& Development, 1, 229-242.

Caron, A., Caron, R., Carlson, V. (1979). Infant perception of the invariant shape of objects varying in slant. Child Development, 50, 716-721.

CoOK, M., \& BIRCH, R. (1984). Infant perception of the shapes of tilted plane forms. Infant Behavior \& Development, 7, 389-402.

Craton, L. G., Yonas, A. (in press). Five-month-old infants' sensitivity to relative motion information for depth at an edge. Child Development.

Fox, R., McDaniels, C. (1982). The perception of biological motion by human infants. Science, 218, 486-487.

Gibson, E. J., a WALker, A. S. (1984). Development of knowledge of visual-tactual affordances of substance. Child Development, 55 , 453-460.

Girson, J. J. (1966). The senses considered as perceptual systems. Boston: Houghton Mifflin.

GIBson, J. J. (1979). The ecological approach to visual perception. Boston: Houghton Mifflin.

Granrud, C. E., Yonas, A., Smith, I., Arterberry, M. E. , GlickSMAN, M. L., \& Sorknes, A. C. (1984). Infants' sensitivity of accretion and deletion of texture as information for depth at an edge. Child Development, 55, 1630-1636.

Horowttz, F. D., PADEN, L., Bhana, K., \& Self, P. (1972). An infantcontrol procedure for studying visual fixations. Developmental Psychology, 7, 90.

Julesz, B. (1971). Foundations of cyclopean vision. Chicago: University of Chicago Press.

Kellman, P. J. (1984). Perception of three-dimensional form by human infants. Perception \& Psychophysics, 36, 353-358.

Kellman, P. J., Hofsten, C. von., \& Soares, J. (1987). Concurrent motion in infant event perception. Infant Behavior \& Development, 10, 1-10. 
Kellman, P. J., \& ShoRT, K. (1985, April). Infant form perception from perspective transformations. Paper presented at the meetings of the Society for Research in Child Development, Toronto.

NAKAYAMA, K., \& LoOMis, J. (1974). Optical velocity patterns, velocitysensitive neurons, and space perception: A hypothesis. Perception, 3, 63-80.

Nanez, J. (1987). Perception of impending collision in 3- to 6-weekold infants. Unpublished doctoral thesis, University of Minnesota.

OWSLEY, C. (1983). The role of motion in infants' perception of solid shape. Perception, 12, 707-717.

Shaw, L., Roder, B., \& BUShNell, E. (1986). Infants' identification of three-dimensional form from transformations of linear perspective. Perception \& Psychophysics, 40, 301-310.
Yonas, A., Arterberry, M. E., \& Granrud, C. E. (1987). Fourmonth-old infants' sensitivity to binocular and kinetic information for three-dimensional object shape. Child Development, 58, 910-917.

Yonas, A., Pettersen, L., Lockman, J. (1979). Young infants sensitivity to optical information for collision. Canadian Joumal o Psychology, 33, 268-276.

Yonas, A., Pick, H. L., JR. (1975). An approach to the study o infant space perception. In L. B. Cohen \& P. Salapatek (Eds.), In fant perception: From sensation to cognition (Vol. 2, pp. 3-32). New York: Academic Press.

(Manuscript received March 12, 1987; revision accepted for publication December 4, 1987.)

\title{
Announcement
}

\author{
13th Annual Boston University Conference on Language Development \\ Boston, Massachusetts \\ October 21, 22, and 23, 1988
}

The 13th Annual Boston University Conference on Language Development is scheduled for October 21, 22, and 23, 1988. The keynote speaker will be Melissa Bowerman, Max Planck Institute of Psycholinguistics, The Netherlands.

Sessions will focus on this year's three main themes: Language Acquisition-theoretical and functional approaches. Literacy-minorities, social class and literacy, teachers as researchers, perspectives on texts and reader/writer relationships, multidisciplinary perspectives on literacy: sociocultural, cognitive, linguistic, educational, anthropological. Second Language Acquisition/Bilingualism-spontaneous second language acquisition, input and markedness, learner strategies and skills.

For a preliminary program, contact the Conference Committee, Conference on Language Development, Boston University, 605 Commonwealth Ave., Boston, MA 02215 (Tel: (617)353-3085). 